\title{
SISTEMA URUBU: A CIÊNCIA CIDADÃ EM PROL DA CONSERVAÇÃO DA BIODIVERSIDADE
}

\section{SISTEMA URUBU: CITIZEN SCIENCE FOR THE CONSERVATION OF THE BIODIVERSITY}

\section{SISTEMA URUBU: LA CIENCIA CIUDADANA EN PROLE DE LA CONSERVACIÓN DE LA BIODIVERSIDAD}

\author{
Érika Paula Castro - erika.castro06@hotmail.com \\ Alex Bager - abager@ecoestradas.org
}

RESUMO: Atropelamento de animais em estradas é um dos impactos mais estudados em Ecologia de Estradas no mundo. Atualmente diversos projetos e plataformas de ciência cidadã têm sido desenvolvidos para quantificar esse impacto, como é o caso do Sistema Urubu, a maior rede para conservação da biodiversidade brasileira. Trata-se de uma plataforma de ciência cidadã para coleta e gestão de dados de atropelamento de fauna selvagem, criada em 2014 pelo Centro Brasileiro de Estudos em Ecologia de Estradas. A principal ferramenta do sistema é um aplicativo, gratuito e disponível para Android e IOs, que conta hoje com mais de 23 mil usuários e mais de 24 mil registros. Seu grande diferencial, quando comparado a outros projetos e plataformas, está na confiabilidade dos dados, uma vez que possui um rigoroso processo de validação de registros realizado por especialistas das diferentes classes de fauna. Além disso, trata-se de uma plataforma que gerou publicação de vários artigos científicos, além de ter contribuído para estruturação da Estratégia Nacional para Mitigação de Impactos da Infraestrutura Viária na Biodiversidade (BioInfra Brasil) e para criação do Projeto de Lei 466/15, já aprovado pela Câmara dos Deputados.

PALAVRAS-CHAVE: Atropelamento de fauna; Ecologia de estradas; Políticas públicas; Tecnologia.

ABSTRACT: Road kills are one of the most studied impacts in Road Ecology in the world. Currently, several projects and platforms for citizen science have been developed to quantify this impact, such as the Urubu System, the largest network for Brazilian biodiversity conservation. This is a citizen science platform for the collection and management of wildlife trampling data, created in 2014 by the Brazilian Center for Studies in Road Ecology (Centro Brasileiro de Estudos em Ecologia de Estradas -

Licença CC BY:

Artigo distribuído sob os termos Creative Commons, permite uso e distribuição irrestrita em qualquer meio desde que o autor credite a fonte original. CBEE). The main tool of the System is an app that is free and available for Android and IOs, that has more than 23 thousand users and more than 24 thousand records. Its great advantage over other projects and platforms is the reliability of its data, as it has a rigorous process of record validation, performed by specialists in the different classes of fauna. It is also a platform that generated scientific publications, as well as contributing to the structuring of the National Strategy for the Mitigation of Impacts of 
Road Infrastructure in Biodiversity (Estratégia Nacional para Mitigação de Impactos da Infraestrutura Viária na Biodiversidade - BioInfra Brazil) and to the creation of Bill 466/15, already approved in the Chamber of Deputies.

KEYWORDS: Road kill; Road ecology; Public policy; Technology.

RESUMEN: Atropellamiento de animales en carreteras es uno de los impactos más estudiados en Ecología de Carreteras en el mundo. Actualmente diversos proyectos y plataformas de ciencia ciudadana han sido desarrolladas para cuantificar este impacto, como es el caso del Sistema Urubu, la mayor red para la conservación de la biodiversidad brasileña. Se trata de una plataforma de ciencia ciudadana para colecta y gestión de datos de atropellamiento de fauna salvaje, creada en 2014 por el Centro Brasileño de Estudios en Ecología de Carreteras. La principal herramienta del Sistema es un aplicativo, gratuito y disponible para Android e IOs, que cuenta hoy con más de 23 mil usuarios y más de 24 mil registros. Su gran diferencial, cuando comparado a otros proyectos y plataformas, está en la confiabilidad de los datos, porque posee un riguroso proceso de validad de registros realizado por especialistas de las diferentes clases de fauna. Además de eso, se trata de una plataforma que generó publicación de varios artículos científicos, además de haber contribuido para la estructuración de la Estrategia Nacional para Mitigación de Impactos en la Infraestructura Viaria en la Biodiversidad (BioInfra Brasil) y para la creación del Proyecto de Ley 466/15, ya aprobado por la Cámara de Deputados.

PALABRAS-CLAVE: Atropellamiento de fauna; Ecología de carreteras; Políticas públicas; Tecnología.

\section{INTRODUÇÃO}

Infraestruturas viárias são fundamentais para o desenvolvimento socioeconômico de um país (FEARNSIDE, 1990; PERZ et al., 2007). Apesar disso, por serem agentes fragmentadores de habitat, seus processos de construção e operação são responsáveis por diversos impactos sobre a biodiversidade local (COFFIN, 2007; LAURANCE; GOOSEM; LAURANCE, 2009). A preocupação com estes impactos levou ao surgimento de um ramo da Ecologia Aplicada, conhecido com Ecologia de Estradas, "Road Ecology" (FORMAN; ALEXANDER, 1998). Este segmento tem ganhado cada vez mais a atenção de pesquisadores, sendo o primeiro trabalho brasileiro publicado em 1998 (NOVELLI; TAKASE; CASTRO, 1998).

Dentre os impactos causados, um dos mais estudados e visível é o atropelamento de fauna (CARVALHO, 2014; JAEGER, 2015). Segundo uma estimativa realizada pelo Centro Brasileiro de Estudos em Ecologia de Estradas (CBEE, 2019), 475 milhões de animais silvestres são mortos ao ano por atropelamento no Brasil.

A forma mais usual de coleta de dados de atropelamento de fauna por muito tempo foi com a realização de projetos de monitoramento sistemático, devido fornecerem dados de alta qualidade. Entretanto, esse tipo de projeto demanda um alto esforço amostral e elevado custo, sendo por isso aplicado para pequenas áreas amostrais (BARTHELMESS; BROOKS, 2010; CARO; HARGEL; STONER, 2000). Por outro lado, para efetiva conservação das espécies e para haja tomada de decisão em termos de medidas de mitigação e políticas públicas, é necessário que haja um volume maior de dados e de um maior espaço de tempo (VAN DER REE et al., 2011; VERCAYIE; HERREMANS, 2015), comumente não obtidos por estes tipos de projetos. 
Atualmente diversos projetos envolvendo a participação ativa dos diferentes segmentos da sociedade tem ganhado a atenção de pesquisadores das diversas áreas do conhecimento, quando é necessário grande volume de dados coletados (CHANDLER et al., 2017; DICKINSON et al., 2012; MONGE-NÁJERA; SEAS, 2018). Apesar de existirem diferentes tipos de projetos de ciência cidadã, dependendo do nível de envolvimento da sociedade, o tipo comumente desenvolvido é o contributivo, que são projetos estruturados por cientistas nos quais a sociedade participa apenas na coleta de dados (DICKINSON et al., 2012; GRAY et al., 2017).

Além da vantagem do aumento significativo de dados coletados, existe a grande escala de abrangência e ainda o baixo investimento em recursos e tempo (GRAY et al., 2017; KOBORI et al., 2016; WILSON et al., 3013). Contudo, há grande discussão no que se refere à confiabilidade dos dados proporcionados por esse tipo de projeto (DICKINSON; ZUCKERBERG; BONTER, 2010; GARDINER, 2012), além do quanto estes propiciam a tomada de decisões (CONRAD; HILCHEY, 2011) e resultam de fato na conservação (GRAY et al., 2017).

No caso de projetos de ciência cidadã para monitoramento de fauna atropelada, quando estes envolvem o uso de aplicativos de celular como forma de coleta de dados, é possível que os mesmos sejam georreferenciados no ato da coleta (HEIGL et al., 2016; VERCAYIE; HERREMANS, 2015). Isso melhora expressivamente a qualidade dos dados, aumentando a precisão e favorecendo para que haja a diminuição dos erros e a padronização da coleta (OLSON et al., 2014). Outro complemento ressaltado por Périquet e colaboradores como sendo de suma importância para melhores resultados nestes projetos refere-se ao envio de uma foto do animal para permitir a identificação posterior da espécie por um especialista.

No Brasil, o Centro Brasileiro de Estudos em Ecologia de Estradas (CBEE) criou em 2014 o que hoje é a maior rede de ciência cidadã para conservação da biodiversidade do país, o Sistema Urubu. O Sistema Urubu foi desenvolvido com a finalidade de mapear os atropelamentos de animais nas rodovias brasileiras, tendo como principal ferramenta um aplicativo de celular para coleta de dados. Seu principal diferencial frente a outras plataformas e aplicativos de mesma finalidade está em um rigoroso processo de identificação de espécies por meio de fotos enviadas pelos usuários, processo este feito por especialistas voluntários das diferentes classes de animais.

O presente artigo tem por objetivo apresentar o Sistema Urubu, suas ferramentas e funcionalidades, bem como compará-lo com outros aplicativos e plataformas de ciência cidadã que possuem a mesma finalidade.

\section{O SISTEMA URUBU E AS SUAS FUNCIONALIDADES}

\section{Objetivo do sistema}

A plataforma foi desenvolvida com o objetivo de reunir registros de atropelamento de animais selvagens, animais vivos cruzando e medidas de mitigação. Entende-se como medidas de mitigação estruturas ou dispositivos ao longo da estrada que atuam direta ou indiretamente como redutores de atropelamentos, tais como placas, dispositivos de redução de velocidade de veículos, passagens de fauna em todo território brasileiro. O Sistema Urubu foi lançado em 2014 com a finalidade de criar uma rede de conservação da biodiversidade. Surgiu devido à necessidade de se ter dados confiáveis, consistentes e atualizados sobre atropelamento de 
animais em todo território nacional. Estes dados possibilitam a tomada de decisão junto aos órgãos governamentais, instituições, concessionárias de rodovias e pesquisadores, além da proposição de políticas públicas e medidas de mitigação.

\section{ENTENDENDO O FUNCIONAMENTO DO SISTEMA}

O Sistema Urubu possui ferramentas utilizadas para coleta e envio de dados, como o aplicativo Urubu Mobile e a Ferramenta de importação; plataformas de gestão, tanto de usuários, feita pelo Portal de Segurança, quanto de registros, feita por meio do Urubu $W e b$; além de possuir a Ferramenta de análise e o Urubu Map, que são plataformas de análise e visualização dos dados, respectivamente.

Por seu caráter de ciência cidadã, o sistema se beneficia da colaboração de voluntários tanto para coleta de registros quanto para identificação dos dados recebidos. São dois tipos de voluntários: (i) Usuários, que são aqueles responsáveis pelo envio de registros (animais atropelados, avistamento de animais vivos cruzando as estradas e as ferrovias ou as estruturas para mitigação de atropelamento de fauna); e (ii) Validadores, que são especialistas ad hoc, cuja função é identificar taxonomicamente o registro, tornando-o padronizado e de maior confiabilidade.

O registro, após ser coletado e enviado via aplicativo mobile (Urubu Mobile) ou pela ferramenta de importação, é direcionado para uma base de dados on-line (Urubu Web), em que passará por um processo de gestão. Este processo envolve quatro fases: Classificação, Avaliação, Validação e Aprovação, que serão detalhadas no segmento "Ferramentas do Sistema”, tópico Urubu Web.

Após identificado o registro, fica disponível para análises (Ferramenta de análise) e visualização geográfica (Urubu Map). A Figura 1 apresenta, de forma simplificada, o funcionamento do sistema. O detalhamento de cada fase será apresentado nos tópicos seguintes. 
FIGURA 1. Fluxo de funcionamento do Sistema Urubu.

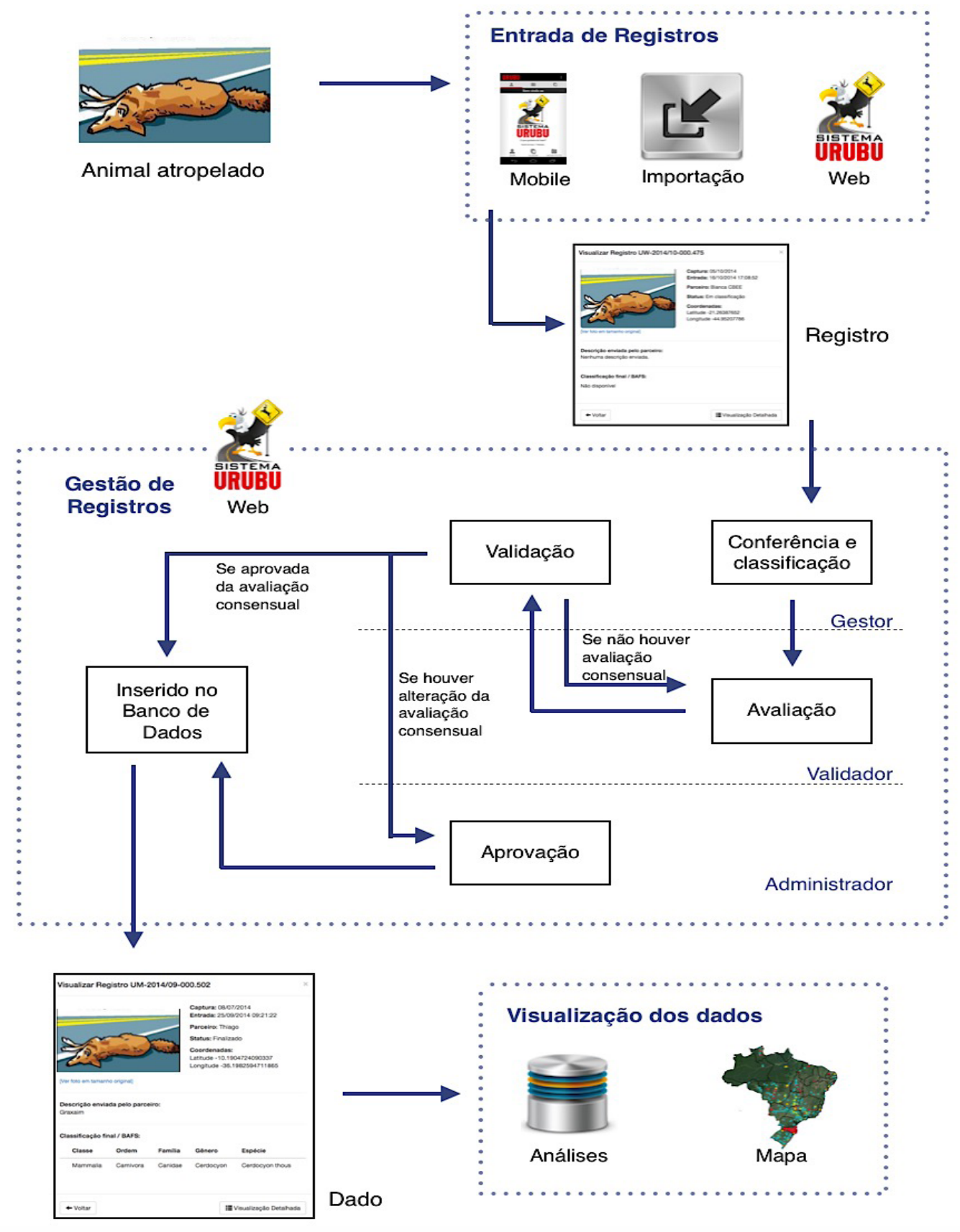

Fonte: Módulo 2-Sistema Urubu (2017)

\section{AS FERRAMENTAS DO SISTEMA}

O Sistema Urubu apresenta diferentes ferramentas que atuam nos processos de coleta e envio dos registros, gerenciamento de cadastros e perfis de acesso, recebimento, gestão e gerenciamento de registros, emissão de relatórios e visualização da distribuição dos dados. São elas: 
Consiste em um aplicativo gratuito, disponibilizado para tablets e smartphones com sistema operacional Android ou iOS, disponível em português, espanhol e inglês. Esse componente é destinado às pessoas interessadas em contribuir com o envio de dados para o sistema e se encontra habilitado para o recebimento de registros em escala mundial.

Na primeira utilização do app é realizado o cadastro, em que um login e uma senha são gerados para que o usuário possa ter acesso ao envio de fotos e também acompanhar o processamento de seus dados, via Urubu $W e b$ (ver a seguir).

O uso do app requer que o GPS dos equipamentos esteja ativado ou o registro por foto estará desabilitado. Após tirar foto do registro e antes de salvá-la, aparecerá para o usuário a opção “descrição", em que é possível o preenchimento com observações pessoais, não sendo obrigatória.

Com o salvamento da foto, o aplicativo registra automaticamente as coordenadas geográficas, o usuário que fez o registro e a data. As fotos tiradas pelo usuário (enviadas ou não ao sistema) permanecem armazenadas no “Álbum”. No caso das fotos já enviadas, um ícone $(\sqrt{ })$ aparecerá no canto inferior esquerdo da imagem, como é mostrado na Figura 2.

O envio dos registros do álbum do aplicativo somente será possível com acesso à internet. Caso o usuário não tenha acesso à internet no momento dos registros, basta que, posteriormente, acesse o álbum de fotos, dentro do próprio aplicativo (onde as fotos se encontram salvas), selecione a foto desejada e a encaminhe.

É permitido o envio de mais de uma foto simultaneamente por meio do aplicativo, entretanto é necessário que o usuário se atente para que não haja o envio de mais de uma foto de um mesmo animal, causando duplicação de registros. Caso isso ocorra, o registro duplicado é excluído pelo gestor e o usuário é comunicado. 
FIGURA 2. Funcionamento das ferramentas do Urubu Mobile para coleta e envio de registros.
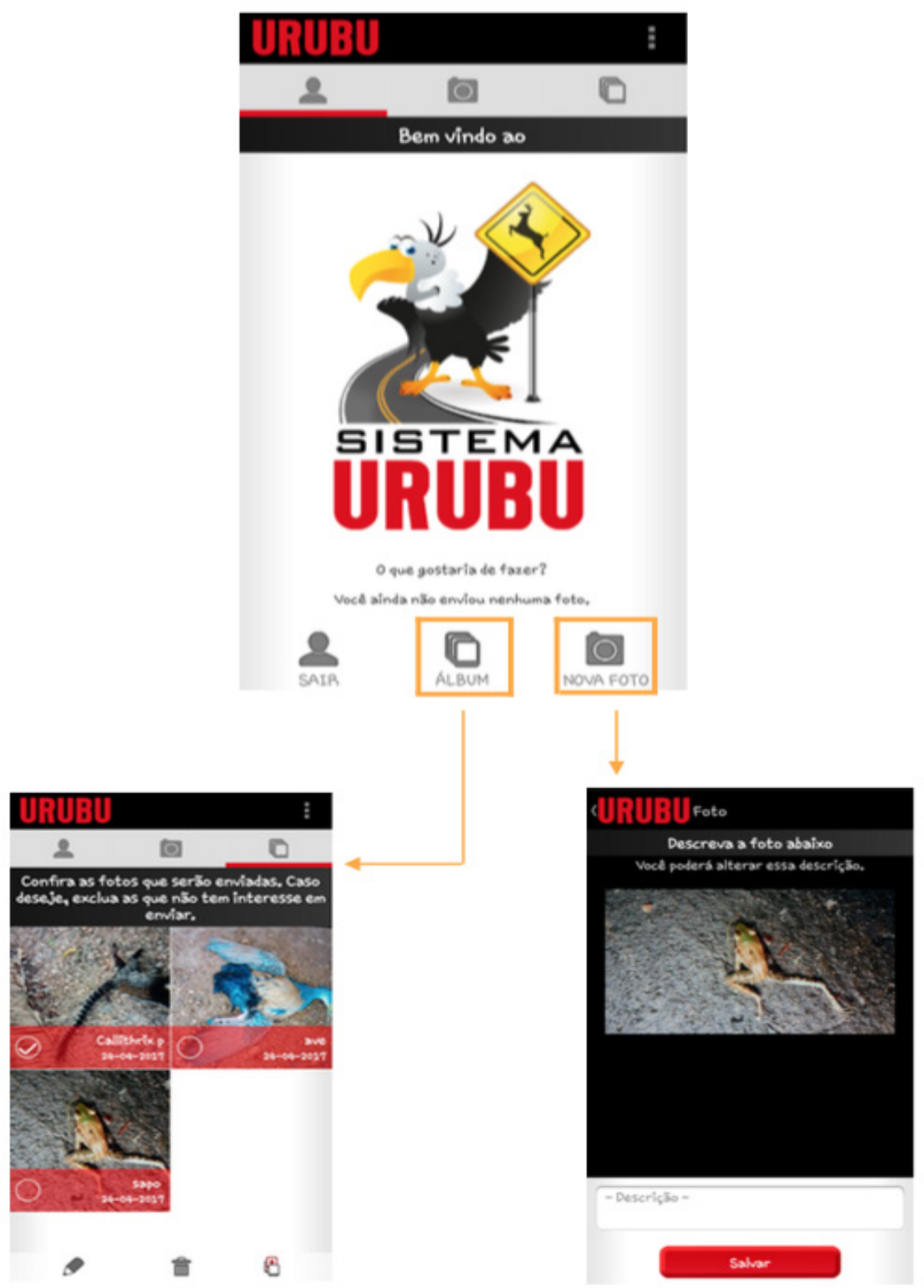

Fonte: Do autor (2019)

URUBU WEB

Todo o processo de identificação e gestão dos dados do sistema é realizado em ambiente web através do Urubu $W e b$, por onde ocorre o recebimento, a inserção, a gestão e a validação dos dados de fauna atropelada e medidas de mitigação, além de outras funcionalidades. Por essa página ocorre toda a gestão de registros e usuários. Ela é segmentada para 3 públicos distintos: (i) usuário do Urubu Mobile, devendo acessar com seu login e sua senha do aplicativo, tendo disponíveis as informações referentes aos registros que ele enviou, tais como envio, aprovação, classificação e validação (que serão descritos a seguir); (ii) validadores, em que terão acesso apenas aos registros referentes à sua classe de atuação (anfíbios, aves, mamíferos ou répteis) e poderão realizar o processo de avaliação 
destes registros; e (iii) gestores e administradores, em que podem fazer a gestão dos registros recebidos e dos usuários e dos validadores do sistema (Figura 3). O acesso a essa plataforma, bem como ao Portal de Segurança, Ferramenta de Importação e Urubu Map (ferramentas que serão descritas posteriormente), ocorre através do link http://bafs.cbee.ufla.br/.

FIGURA 3. Realização do acesso no Urubu Web e visualização de dados enviados.
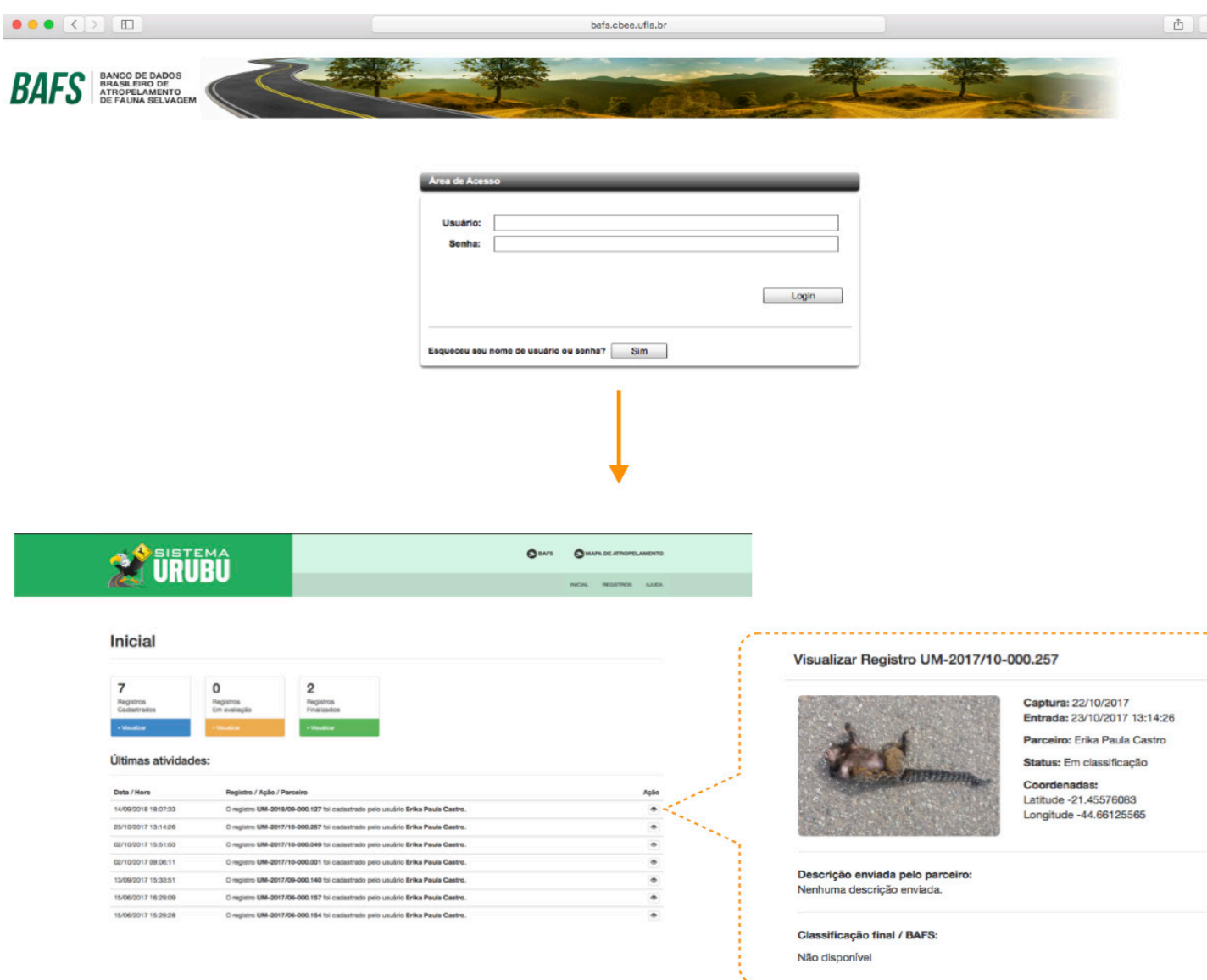

Fonte: Urubu Web(2019)

Como comentado, é por meio do Urubu Web que é feita a classificação dos dados por especialistas ad hoc voluntários, os validadores. Somente é cadastrado como validador aquele especialista que é aprovado pelo gestor do sistema.

Para classificação dos registros, existe uma base taxonômica atualizada das quatro classes de vertebrados silvestres (anfíbios, aves, mamíferos e répteis), o que garante que os dados serão classificados de forma padronizada e respeitando as mudanças taxonômicas. No caso das medidas de mitigação, estas passam apenas pela aprovação e classificação do gestor do sistema, não sendo estas enviadas aos validadores.

Para estarem aptos a serem inseridos no banco de dados do Sistema Urubu e liberados para visualização da sociedade, os dados passam por 4 etapas:

1. Classificação: Esta etapa é realizada pelo gestor do sistema. Após o envio do registro, o mesmo é encaminhado para uma base de dados dentro do Urubu Web. O gestor analisa a fotografia, 
as coordenadas do registro e o identifica a nível de classe. Para que o mesmo seja aprovado e classificado, é necessário que atenda os seguintes requisitos: (i) tratar-se de um animal atropelado em uma estrada, um animal vivo cruzando a estrada ou uma medida de mitigação; (ii) ser uma foto tirada pelo aplicativo, não são aceitas fotocópias; (iii) possuir uma coordenada que esteja localizada sobre uma estrada, rodovia ou ferrovia, independente de ser área urbana ou não. Quando o registro não possui coordenada que atenda essas exigências, o gestor entra em contato com o usuário para tentar entender se foi um erro da geolocalização (do GPS) ou se trata de um registro incorreto; (iv) não ser um registro duplicado. Alguns usuários tiram mais de uma foto de um mesmo animal e, ao passar pela avaliação do gestor, apenas uma delas é aceita. Ainda, ocorrem casos de dois usuários distintos avistarem e registrarem um mesmo animal. Neste caso, apenas o primeiro registro enviado é contabilizado e aceito. Caso não atenda aos pré-requisitos anteriores, o registro é excluído e uma notificação é enviada ao usuário explicando o motivo para tal exclusão.

2. Avaliação: Todos os registros classificados ficam disponíveis para que os validadores da respectiva classe possam classificá-los. A confiabilidade e o detalhamento das informações do registro são o maior diferencial do Sistema Urubu e para isso existem algumas regras de avaliação: (i) Cada registro pode ser avaliado por até 5 validadores, sendo estes os cinco primeiros que o acessaram e avaliaram; (ii) para que o registro passe para a etapa de validação, é necessário que haja um consenso de, no mínimo, 3 validadores, tendo alcançado o máximo nível taxonômico possível; (iii) registros que atingirem o consenso com 3 validadores no maior nível taxonômico não precisam passar pela validação dos dois outros; (iv) nenhum validador tem acesso à avaliação dos demais validadores. Sempre que novos registros são liberados pelo gestor para passarem para o processo de avaliação, os validadores são automaticamente avisados. Para o gestor, todos os registros em avaliação são visíveis, contudo não estão disponíveis para identificação.

3. Validação: Realizada a avaliação, o gestor verifica se o resultado obtido pelo processo foi suficiente para considerar o registro de um dado válido. Caso um registro, após diversas tentativas de avaliação, não obtenha consenso de 3 validadores, o gestor pode validá-lo considerando o nível taxonômico "classe". Os registros que obtiverem consenso são validados e aprovados automaticamente, sendo inseridos assim no banco de dados e ficando disponíveis para visualização no Urubu Map. Os registros que necessitarem validação do gestor por não obterem consenso passam ainda pela fase de aprovação.

4. Aprovação: Após ter passado pelas 3 etapas anteriores, o registro chega para aprovação do administrador. O mesmo recebe a listagem completa da informação do registro, contendo: a classificação do gestor, as avaliações e a validação (diferente da consensual). É de responsabilidade do administrador realizar a aprovação final, indo de acordo com a avaliação consensual, com a nova classificação realizada pelo gestou ou, ainda, realizar sua própria avaliação. Após aprovado, o registro se torna um dado validado, é inserido no banco de dados e fica disponível para visualização no Urubu Map.

\section{PORTAL DE SEGURANÇA}

O Portal de Segurança é uma ferramenta de gestão de usuários, na qual é possível realizar o cadastro de perfis de acesso e o controle de usuários, projetos e instituições. O sistema possui diferentes categorias de usuários, e cada um deles tem funções e permissões no Urubu $W e b$ distintas. A partir dele, são liberadas estas permissões. A seguir são descritas as categorias de perfis de acesso: 
- Usuário Urubu Mobile: Responsável pelo envio das fotos ao sistema através do aplicativo Urubu Mobile e, para isso, deve fazer seu download e cadastro no aplicativo;

- Validador: Responsável pela identificação dos registros enviados pelos usuários até a classificação taxonômica mais precisa possível via Urubu Web. Cada validador possui dentro do Urubu Web acesso apenas aos registros disponíveis para avaliação da sua respectiva categoria;

- Gestor: Responsável pela gestão dos dados no Urubu Web, realizando uma avaliação prévia dos registros recebidos no sistema e a validação dos dados classificados pelos validadores. Também gerencia as demais ferramentas do sistema, tais como o Portal de Segurança, Urubu Map, entre outros que serão ainda descritos;

- Administrador: Detentor de todos as atribuições do gestor, além de ser responsável pelo controle de todos os processos do sistema, como cadastros e exclusões, e das permissões de cada um dos papéis dentro do Urubu Web e Urubu Map.

Apesar de o Urubu Web encontrar-se disponível para todos os perfis de usuários, como detalhado anteriormente, cada usuário possui diferentes permissões dentro do sistema, como apresentado na Tabela 1.

TABELA 1. Permissões de acesso aos registros no Urubu $W e b$ de acordo com os perfis de usuários.

\begin{tabular}{|c|c|c|c|c|}
\hline \multirow[b]{2}{*}{ Ação } & \multicolumn{4}{|c|}{ Perfis de Usuário } \\
\hline & Administrador & Gestor & Validador & $\begin{array}{l}\text { Usuário Urubu } \\
\text { Mobile }\end{array}$ \\
\hline Criar & Sim & Sim & Não & Não \\
\hline Visualizar & Sim & Sim & $\begin{array}{l}\text { Apenas dados públicos ou } \\
\text { disponíveis para serem } \\
\text { classificados }\end{array}$ & $\begin{array}{l}\text { Apenas dados } \\
\text { próprios }\end{array}$ \\
\hline Atualizar & Sim & Sim & Não & Não \\
\hline Remover & Sim & $\begin{array}{l}\text { Apenas } \\
\text { envio para } \\
\text { lixeira }\end{array}$ & Não & Não \\
\hline
\end{tabular}

Fonte: Do autor (2019)

Como citado, por meio do Portal de Segurança do Sistema Urubu também é realizado o cadastro de usuários, de instituições e de projetos. Uma instituição pode ter um ou mais projetos, e cada projeto pode ter um ou mais usuários. Na realização de cadastro de instituições, uma vez que a mesma está cadastrada, o sistema permite que usuários e projetos possam ser vinculados a ela. Se esta apresentar diversos pontos de monitoramento, terão seus dados diferenciados a partir de cadastros de diferentes projetos.

Cada instituição e cada projeto podem ter diversos usuários vinculados. Sendo assim, ao serem enviados dados, eles são armazenados diretamente em seu respectivo projeto. Os usuários são vinculados a perfis de acesso que determinam as funcionalidades e os dados que podem acessar, alterar e deletar, ou seja, a instituição à qual o usuário está vinculado pode definir quais informações 
e projetos o mesmo terá acesso em sua conta. Para quaisquer categorias de acesso, exceto do usuário Urubu Mobile, o cadastro é realizado por meio do preenchimento de um formulário, como ilustrado na Figura 4. Este formulário é preenchido pelo gestor e distingue-se conforme o perfil do cadastro. No caso do Usuário Urubu Mobile, o cadastro é realizado por meio do próprio aplicativo, no primeiro acesso, através da opção "Entrar/Cadastrar" na tela inicial.

FIGURA 4. Formulário para realização de cadastro de usuários.

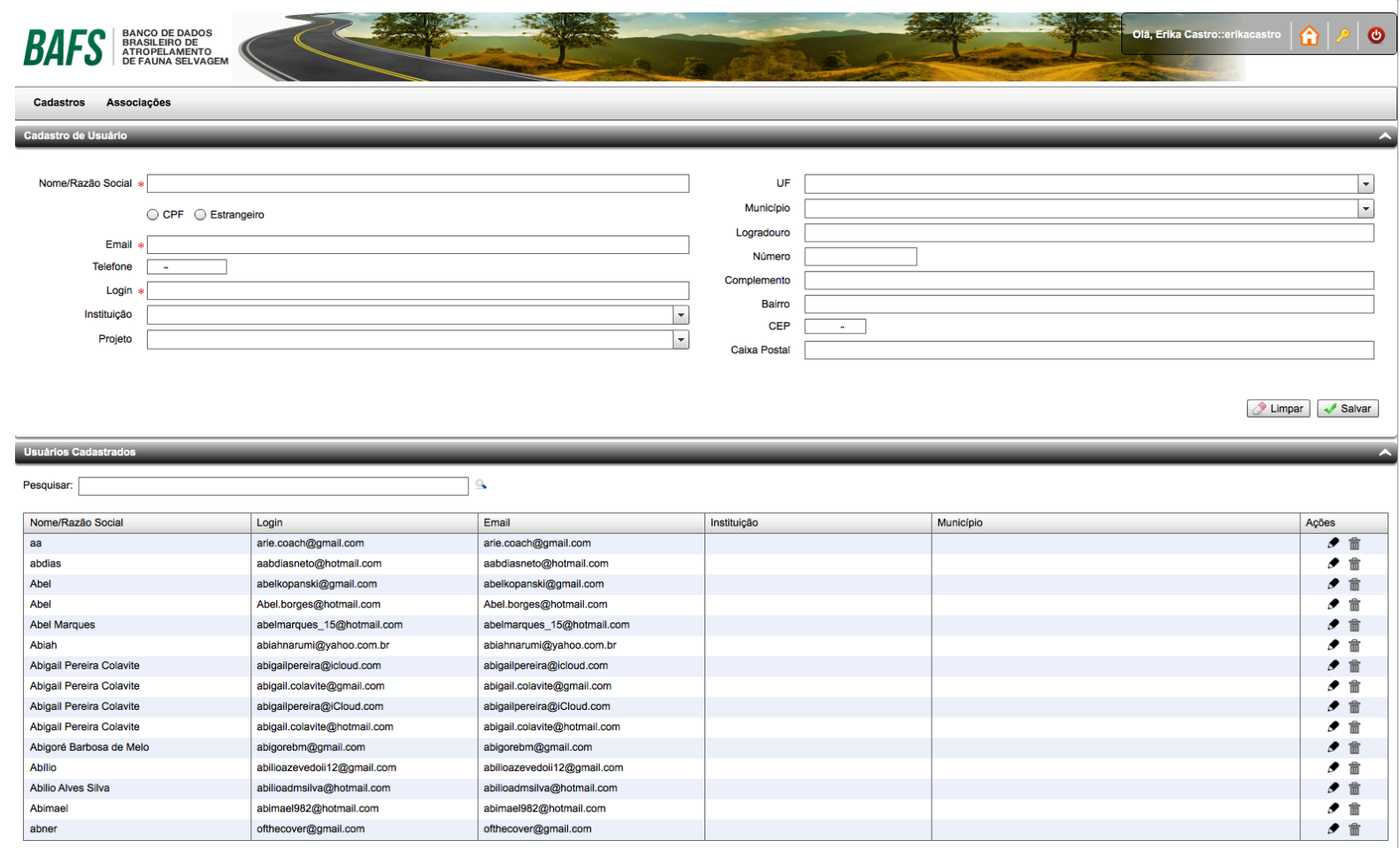

\begin{tabular}{|l|l|l|l|l|l|l|l|l|l|l|l|l|l|}
\hline \multirow{2}{*}{} & $<$ & 1 & 2 & 3 & 4 & 5 & 6 & 7 & 8 & 9 & 10 & 7 & 8 \\
\hline
\end{tabular}

Fonte: Urubu Web (2019)

Um formulário similar é utilizado para vincular usuários a projetos e/ou instituições. No caso de vinculação a projetos, deve ser informado o período de desenvolvimento do projeto, sua origem, a quilometragem amostrada da malha viária, o tipo de monitoramento (a pé ou carro), tipo de pavimento, número de pistas e presença ou ausência de acostamento. Caso seja necessário, é permitido ao administrador cadastrar novos perfis de acesso ao sistema e vincular instituições a esses perfis.

\section{FERRAMENTA DE IMPORTAÇÃO}

Esta ferramenta permite que seja inserido um grande número de dados enviados por parceiros. A inserção ocorre por meio de uma planilha de importação em Excel e tem por objetivo facilitar que projetos ou empresas que tenham interesse em adotar o uso do Sistema Urubu possam fazê-lo de forma mais ágil. Este processo é realizado pelo gestor, que verificará que os dados recebidos estejam adequados ao banco de dados (tamanho de fotos, taxonomia e localização corretas). Por padronização da equipe do Sistema Urubu, todos os dados que possuem foto são encaminhados aos validadores. Esta regra visa garantir confiabilidade dos dados. Em caso de registros que não apresentam imagens, a inserção é feita diretamente no banco de dados e no Urubu Map, sendo então classificados como "Dados não validados”. 


\section{FERRAMENTA DE ANÁLISE}

A ferramenta de análise é o meio pelo qual é feita a emissão de relatórios de análises dos dados inseridos no sistema. Para possibilitar tais análises, conta com filtros em que é possível restringir a busca de dados por taxonomia, estado, unidade de conservação, dentre outras informações dos registros, conforme a necessidade. Esta é uma ferramenta disponível apenas para administrador e gestores.

\section{URUBU MAP}

O Urubu Mapé um sistema de visualização dos registros (Figura 5). A partir deste componente, é possível visualizar os dados inseridos no sistema, após sua aprovação, de acordo com a sua localização. No mapa encontram-se opções de visualização, filtros para visualização dos dados, opções de zoom, navegação e escala, como ilustrado na Figura 5.

FIGURA 5. Urubu Map e aplicações disponíveis para visualização dos dados.

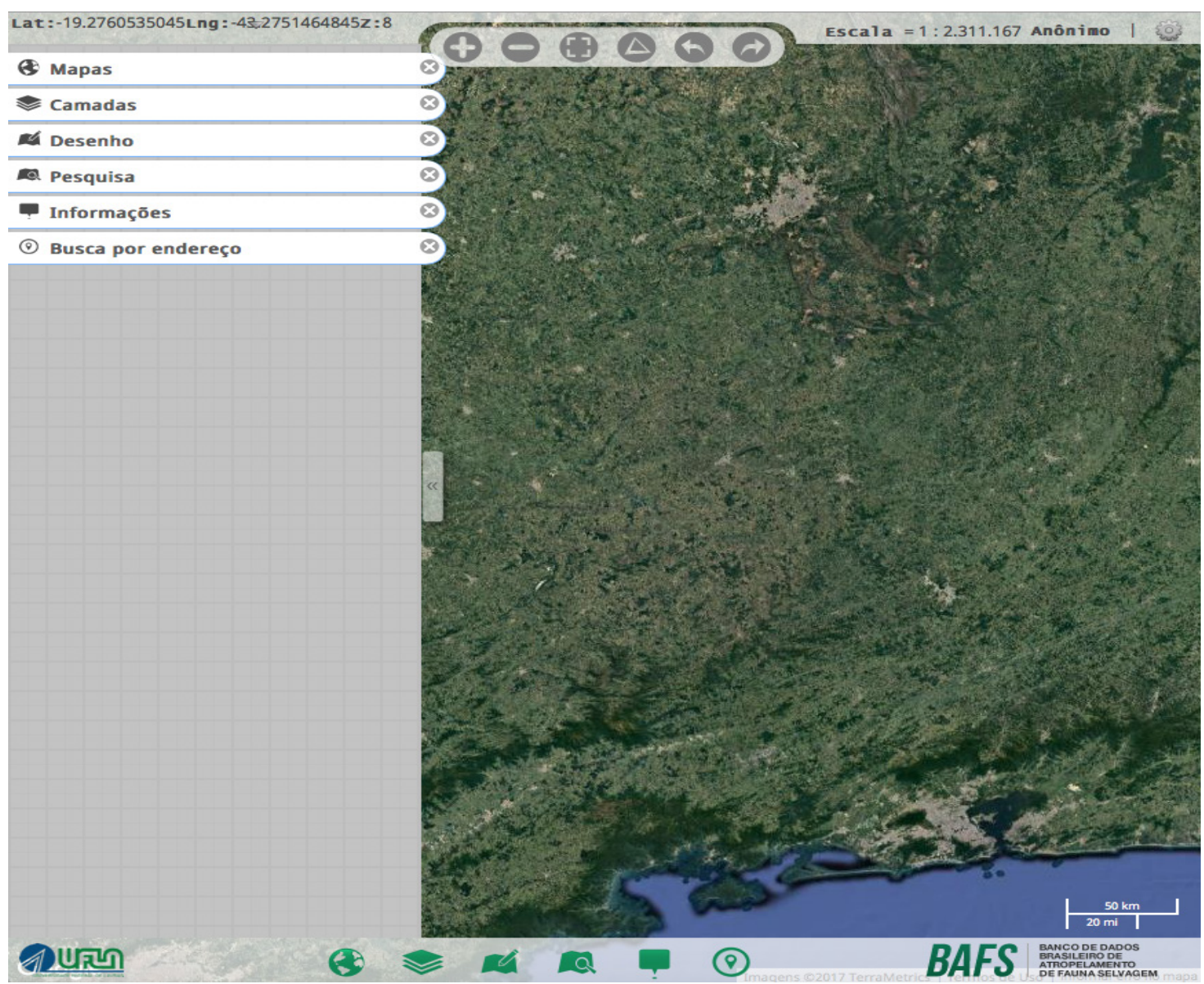

Fonte: Urubu Map (2019)

Após ser aprovado para o banco de dados do sistema, o registro é inserido automaticamente no mapa, como apresentado na Figura 6. Cada cor representa uma classe de animais (anfíbio, ave, mamífero ou réptil). Ao selecionar algum ponto, é possível que o usuário visualize o detalhamento do respectivo registro, com acesso a informações como: fotografia, projeto vinculado, tipo de coleta de dados (eventual ou sistemática), empreendimento (estrada ou ferrovia), usuário cedente dos dados e classificação taxonômica do espécime. 
FIGURA 6. Disposição dos dados do sistema por classe no Urubu Map.

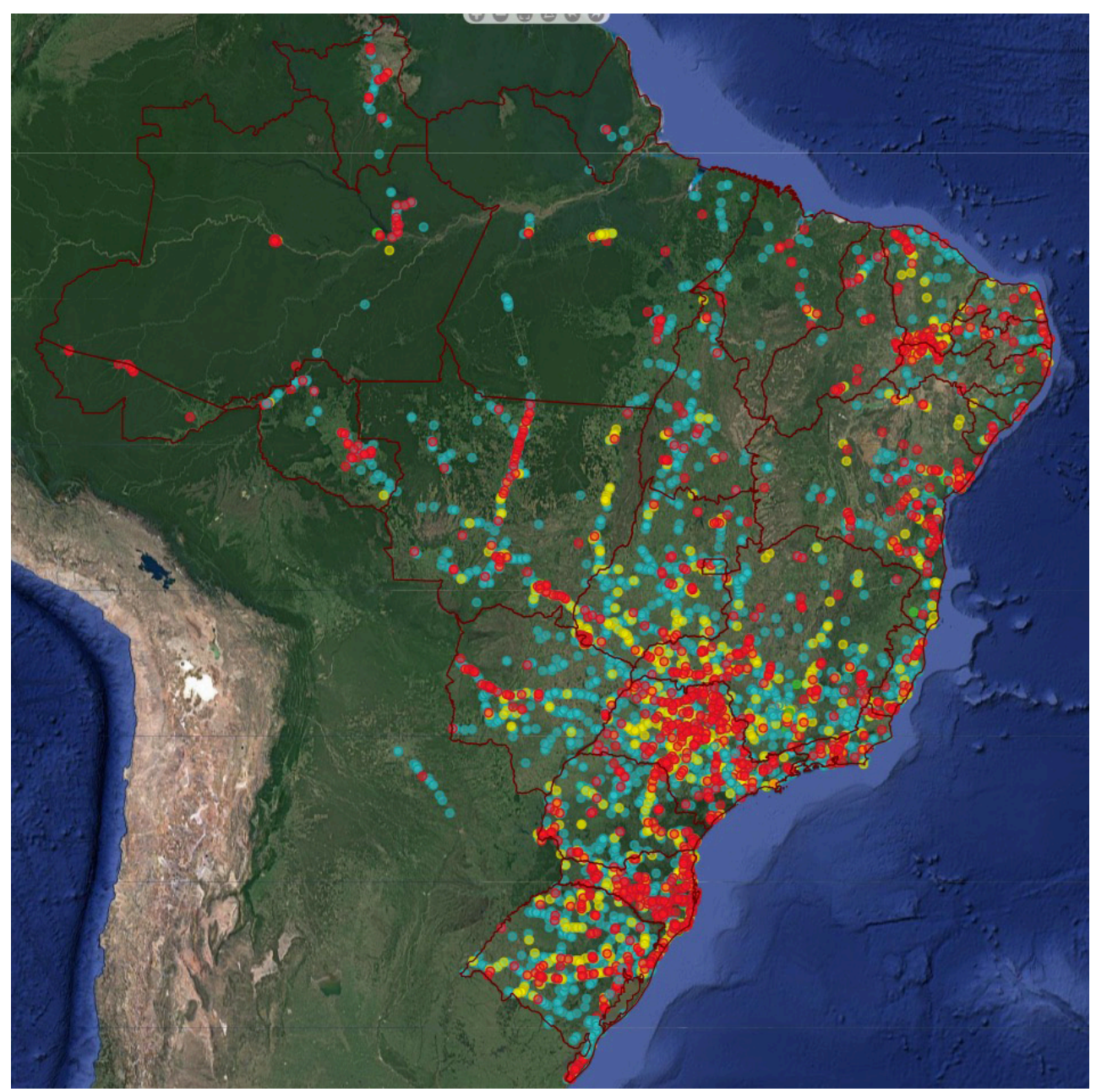

Fonte: Urubu Map (2019)

\section{A EVOLUÇÃO DO SISTEMA URUBU}

O Sistema Urubu, apesar de ter sido lançado em 2014, levou um tempo para que se tornasse a maior rede brasileira para conservação da biodiversidade. Atualmente o sistema conta com mais de 24 mil registros enviados e cerca de 23 mil usuários, o que demandou grande investimento em divulgação por meio das redes sociais e da mídia. O Gráfico 1 mostra o progresso anual referente ao número cadastros de usuários desde o seu lançamento.

Apesar de ser um projeto de importante cunho ambiental, por meio dos números é possível observar que houve alguns momentos de maior captura e envolvimento de usuários. Dois momentos de grande importância foram o lançamento do Aplicativo, em 2014, que atraiu de maneira significativa a mídia e levou a um número de novos cadastros, superando 7000. Além disso, em 2015 foi realizada uma reportagem pelo Fantástico, que impactou positivamente com a captação de mais de 5000 usuários, sendo este o ano de maior destaque, com quase 10 mil novos cadastros. 
GRÁfICO 1. Progresso anual do cadastro de novos usuários no Sistema Urubu desde seu lançamento em 2014.

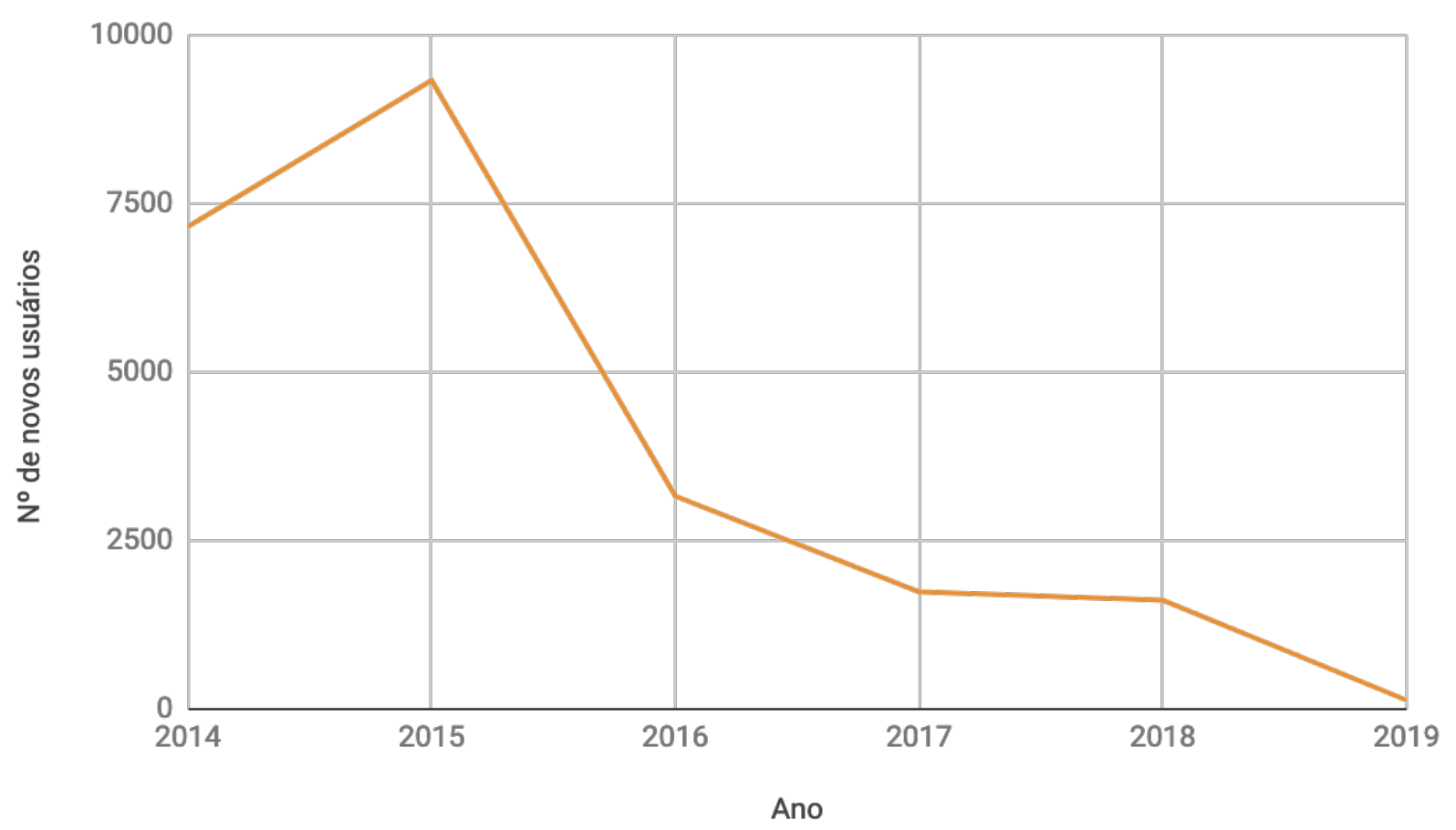

Fonte: Do autor (2019)

Como forma de engajar e ter mais envolvimento dos usuários, entre os anos de 2014 e 2017 foram produzidos infográficos mensais para apresentar as principais ações realizadas e o progresso do sistema. Este era um meio de destacar usuários e validadores mais participativos, além de apresentar as principais espécies identificadas. Além do infográfico, outra estratégia de reconhecimento utilizada é a certificação dos principais validadores mensais.

Os dados obtidos pelo Sistema Urubu propiciaram a produção de diversos artigos científicos referentes ao atropelamento de fauna em estradas brasileiras e distribuição de espécies no país (CULOT et al., 2019; PINTO et al., 2018; GRILO et al., 2018). Além disso, o sistema foi fundamental para criação da Estratégia Nacional para Mitigação de Impactos da Infraestrutura Viária na Biodiversidade (BioInfra Brasil), uma iniciativa semelhante a um Plano de Ação Nacional, elaborada pelo CBEE junto a outras instituições. Seu objetivo é melhorar o conhecimento dos impactos das infraestruturas viárias sobre a biodiversidade e alavancar políticas públicas para mitigação destes impactos.

Outro importante resultado já alcançado refere-se à aprovação na Câmara dos Deputados do PL 466/15, Projeto de Lei desenvolvido em 2015, que "Dispõe sobre a adoção de medidas que assegurem a circulação segura de animais silvestres no território nacional, com a redução de acidentes envolvendo pessoas e animais nas estradas, rodovias e ferrovias brasileiras”. É possível acompanhar o processo de aprovação do projeto por meio do link: https://www.camara.leg.br/ proposicoesWeb/fichadetramitacao?idProposicao $=949094$. 


\section{SISTEMA URUBU FRENTE A OUTRAS PLATAFORMAS}

Em Ecologia de Estradas várias iniciativas têm sido desenvolvidas com o intuito de conhecer, reduzir e mitigar os impactos causados pelas estradas sobre a biodiversidade. Assim como o Sistema Urubu, outros projetos e plataformas de ciência cidadã foram criados para facilitar este estudo em todo o mundo.

Realizou-se uma pesquisa sobre estas plataformas e suas respectivas características comparadas ao Sistema Urubu. Ao todo, foram reunidos 14 projetos/plataformas e as características avaliadas corresponderam ao país do projeto, sistemas operacionais disponíveis (Android, IOs e/ou Web-no caso de não possuir aplicativo), cadastro de usuários (se é necessário ou não realizar cadastro antes do envio de dados), validação de dados (se os registros passavam por um processo de conferência ou classificação taxonômica) e formas de envio de registros.

A maioria dos projetos avaliados apresenta um aplicativo de coleta de dados, entretanto vários deles possibilita também a inserção de registros por meio de formulários on-line. Em apenas três deles não é necessário realizar um cadastro antes de colaborar com o envio de registros e em nenhum deles foram encontradas informações referentes ao processo de validação de registros. Os resultados encontram-se na Tabela 2. 
TABELA 2. Plataformas de ciência cidadã usadas para conhecer os impactos das estradas sobre a biodiversidade. Características referentes ao país do projeto/plataforma, sistemas operacionais disponíveis, cadastro de usuários, processo de validação dos registros e formas de coleta. (Continua)

\begin{tabular}{|c|c|c|c|c|c|}
\hline & País & $\begin{array}{l}\text { Sistema } \\
\text { Operacional }\end{array}$ & $\begin{array}{l}\text { Cadastro } \\
\text { de }\end{array}$ & $\begin{array}{l}\text { Validação } \\
\text { dos dados }\end{array}$ & $\begin{array}{l}\text { Formas de } \\
\text { coleta de dados }\end{array}$ \\
\hline $\begin{array}{l}\text { Animals under } \\
\text { the wheels }\end{array}$ & Bélgica & $\begin{array}{l}\text { Android e } \\
\text { iOs }\end{array}$ & Sim & $\begin{array}{l}\text { Não } \\
\text { informada }\end{array}$ & $\begin{array}{l}\text { Aplicativo e } \\
\text { formulário on- } \\
\text { line }\end{array}$ \\
\hline $\begin{array}{l}\text { Califórnia } \\
\text { Roadkill } \\
\text { Observation } \\
\text { (CROS) }\end{array}$ & $\begin{array}{l}\text { Estados } \\
\text { Unidos }\end{array}$ & $W e b$ & Sim & $\begin{array}{l}\text { Não } \\
\text { informada }\end{array}$ & $\begin{array}{l}\text { Formulário on- } \\
\text { line }\end{array}$ \\
\hline Delta Road Kill & Itália & $W e b$ & Sim & $\begin{array}{l}\text { Não } \\
\text { informada }\end{array}$ & $\begin{array}{l}\text { Apenas por } \\
\text { formulário on- } \\
\text { line }\end{array}$ \\
\hline $\begin{array}{l}\text { Fauna Silvestre } \\
\text { en Carreteras de } \\
\text { Costa Rica }\end{array}$ & $\begin{array}{l}\text { Costa } \\
\text { Rica }\end{array}$ & $W e b$ & Sim & $\begin{array}{l}\text { Não } \\
\text { Informada }\end{array}$ & $\begin{array}{l}\text { Apenas por } \\
\text { formulário on- } \\
\text { line }\end{array}$ \\
\hline $\begin{array}{l}\text { Maine Audubon } \\
\text { Wildlife Road } \\
\text { Watch }\end{array}$ & $\begin{array}{l}\text { Estados } \\
\text { Unidos }\end{array}$ & $W e b$ & Sim & $\begin{array}{l}\text { Não } \\
\text { informada }\end{array}$ & $\begin{array}{l}\text { Apenas por } \\
\text { formulário on- } \\
\text { line }\end{array}$ \\
\hline $\begin{array}{l}\text { Mammals on } \\
\text { roads }\end{array}$ & $\begin{array}{l}\text { Reino } \\
\text { Unido }\end{array}$ & $\begin{array}{l}\text { Android e } \\
\text { iOs }\end{array}$ & Sim & $\begin{array}{l}\text { Não } \\
\text { informada }\end{array}$ & $\begin{array}{l}\text { Aplicativo e } \\
\text { formulário on- } \\
\text { line }\end{array}$ \\
\hline Mam'route & França & $\begin{array}{l}\text { Android e } \\
\text { iOs }\end{array}$ & Sim & $\begin{array}{l}\text { Não } \\
\text { informada }\end{array}$ & $\begin{array}{l}\text { Aplicativo e } \\
\text { formulário on- } \\
\text { line }\end{array}$ \\
\hline
\end{tabular}


TABELA 2. Plataformas de ciência cidadã usadas para conhecer os impactos das estradas sobre a biodiversidade. Características referentes ao país do projeto/plataforma, sistemas operacionais disponíveis, cadastro de usuários, processo de validação dos registros e formas de coleta. (Conclusão)

\begin{tabular}{|c|c|c|c|c|c|}
\hline & País & $\begin{array}{c}\text { Sistema } \\
\text { Operacional }\end{array}$ & $\begin{array}{c}\text { Cadastro } \\
\text { de } \\
\text { usuários }\end{array}$ & $\begin{array}{l}\text { Validação } \\
\text { dos dados }\end{array}$ & $\begin{array}{c}\text { Formas de } \\
\text { coleta de dados }\end{array}$ \\
\hline Project Splatter & $\begin{array}{l}\text { Reino } \\
\text { Unido }\end{array}$ & Android & Não & $\begin{array}{l}\text { Nāo } \\
\text { informada }\end{array}$ & $\begin{array}{l}\text { Aplicativo, rede } \\
\text { social, } e \text {-mail e } \\
\text { formulário on- } \\
\text { line }\end{array}$ \\
\hline Recosfa & Colômbia & Android & Sim & $\begin{array}{c}\text { Não } \\
\text { Informada }\end{array}$ & $\begin{array}{l}\text { Apenas por } \\
\text { aplicativo }\end{array}$ \\
\hline $\begin{array}{c}\text { Reptile Road } \\
\text { Mortality } \\
\text { (TaiRoN) }\end{array}$ & Taiwan & Web & Não & Sim & $\begin{array}{l}\text { Apenas por } \\
\text { facebook }\end{array}$ \\
\hline $\begin{array}{c}\text { Roadkill - } \\
\text { SPOTTERON }\end{array}$ & Áustria & $\begin{array}{l}\text { Android e } \\
\text { iOs }\end{array}$ & Sim & $\begin{array}{l}\text { Não } \\
\text { informada }\end{array}$ & $\begin{array}{l}\text { Aplicativo e } \\
\text { formulário on- } \\
\text { line }\end{array}$ \\
\hline $\begin{array}{l}\text { Roadkill } \\
\text { radar }\end{array}$ & Não & $\begin{array}{l}\text { Android e } \\
\text { iOs }\end{array}$ & Não & $\begin{array}{l}\text { Não } \\
\text { informada }\end{array}$ & $\begin{array}{l}\text { Apenas por } \\
\text { aplicativo }\end{array}$ \\
\hline $\begin{array}{c}\text { Road Watch } \\
\text { BC }\end{array}$ & Canadá & $\begin{array}{l}\text { Android e } \\
\text { iOs }\end{array}$ & Sim & $\begin{array}{l}\text { Não } \\
\text { informada }\end{array}$ & $\begin{array}{l}\text { Aplicativo e } \\
\text { formulário on-line }\end{array}$ \\
\hline $\begin{array}{l}\text { Sistema } \\
\text { Urubu }\end{array}$ & Brasil & $\begin{array}{l}\text { Android e } \\
\text { iOs }\end{array}$ & Sim & $\operatorname{Sim}$ & $\begin{array}{l}\text { Apenas por } \\
\text { aplicativo }\end{array}$ \\
\hline Sražená zvěr̆ & $\begin{array}{c}\text { República } \\
\text { Checa }\end{array}$ & Android & Sim & $\begin{array}{l}\text { Não } \\
\text { informada }\end{array}$ & $\begin{array}{c}\text { Aplicativo e } \\
\text { formulário on-line }\end{array}$ \\
\hline
\end{tabular}

CONSIDERAÇÕES FINAIS

O Sistema Urubu apresenta ferramentas que trabalham interligadas, garantindo sua eficiência. A principal delas é o aplicativo Urubu Mobile, que permite o envio de registros de atropelamento de fauna silvestre. Todo o processo de gestão feito após o recebimento dos dados garante que não haja duplicação de registros e que estes passem por um processo rigoroso de validação.

Como ferramenta de conservação e participação social, o sistema atua de forma eficaz, uma vez que apresenta constante envolvimento de usuários e conta com confiabilidade nos dados coletados. O sistema propiciou geração de produção científica e favoreceu execução de ações, como a BioInfra Brasil e políticas públicas, com o intuito de reduzir os impactos causados pelas estradas sobre a fauna. 
Existem diversos aplicativos e plataformas de ciência cidadã para coleta de registros de atropelamento de fauna no mundo. Entretanto, por se tratarem de projetos que contam com a colaboração de pessoas, em sua maioria, leigas no assunto, torna-se necessário um processo de refinamento destes dados. Não foram encontradas informações a respeito da validação de registros nos projetos avaliados.

Uma situação que agrava a falta de validação refere-se ao fato de em vários dos aplicativos o envio de foto do animal não ser obrigatório, isso ocorre principalmente quando o dado é enviado ou inserido via formulário on-line, e-mail ou redes sociais. Neste caso, impossibilita inclusive que um processo de validação seja aplicado futuramente nos dados já coletados.

Outro ponto se refere às coordenadas geográficas dos registros, já que quase todos os projetos avaliados permitem o envio de registros por meio de formulários on-line ou outros meios, como e-mail e redes sociais. Em vários dos casos basta que os usuários selecionem em um mapa o ponto onde ocorreu o atropelamento. Para que se tenham dados consistentes para tomada de decisão, é essencial que as informações de coordenadas do atropelamento sejam precisas, o que é inviabilizado por este processo.

Um grande diferencial do Sistema Urubu frente aos projetos e às plataformas levantados neste artigo está na confiabilidade dos dados. O sistema conta com um rigoroso processo de validação para classificação de registros, feito exclusivamente por especialistas. A única maneira de os usuários registrarem e enviarem registros é via aplicativo, já que por meio dele o registro é automaticamente georreferenciado. Esses processos e essas regras garantem dados confiáveis, possibilitando usá-los para tomada de decisão, proposição de políticas públicas e conhecimentos dos hotsposts de atropelamento.

\section{REFERÊNCIAS BIBLIOGRÁFICAS}

BAGER, A. et al. Os caminhos da conservação da biodiversidade brasileira frente aos impactos da infraestrutura viária. Biodiversidade Brasileira, n. 1, p. 75-86, 2016.

BARTHELMESS, E. L.; BROOKS, M. S. The influence of body-size and diet on road-kill trends in mammals. Biodiversity and Conservation, v. 19, n. 6, p. 1611-1629, 2010.

CARO, T. M.; SHARGEL, J. A.; STONER, C. J. Frequency of medium-sized mammal road kills in an agricultural landscape in California. The American Midland Naturalist, v. 144, n. 2, p. 362-370, 2000.

CARVALHO, C. F. et al. Atropelamento de vertebrados, hotspots de atropelamentos e parâmetros associados, BR-050, trecho Uberlândia-Uberaba. 2014.

CBEE. CENTRO BRASILEIRO DE ESTUDOS EM ECOLOGIA DE ESTRADAS. BRASIL: Atropelamentos de fauna selvagem. Lavras: CBEE/UFLA. Disponível em <http://cbee.ufla.br/portal/ atropelometro/> (site atropelômetro). Acesso em: Fevereiro, 2019.

CBEE - CENTRO BRASILEIRO DE ESTUDOS EM ECOLOGIA DE ESTRADAS. Sistema Urubu. Módulo 2 - Sistema Urubu. 2 ed., 11 p., 2017.

CBEE - CENTRO BRASILEIRO DE ESTUDOS EM ECOLOGIA DE ESTRADAS. Sistema Urubu. Módulo 4 - Urubu Web. 2 ed., 23 p, 2017.

CHANDLER, M. et al. Contribution of citizen science towards international biodiversity monitoring. Biological Conservation, v. 213, p. 280-294, 2017. 
COFFIN, A. W. From roadkill to road ecology: a review of the ecological effects of roads. Journal of transport Geography, v. 15, n. 5, p. 396-406, 2007.

CONRAD, C. C.; HILCHEY, K. G. A review of citizen science and community-based environmental monitoring: issues and opportunities. Environmental monitoring and assessment, v. 176, n. 1-4, p. 273-291, 2011.

CULOT, L. et al. ATLANTIC-PRIMATES: a dataset of communities and occurrences of primates in the Atlantic Forests of South America. Ecology, v. 100, n. 1, p. e02525, 2019.

DICKINSON, J. L.; ZUCKERBERG, B.; BONTER, D. N. Citizen science as an ecological research tool: challenges and benefits. Annual review of ecology, evolution, and systematics, v. 41, p. 149-172, 2010.

DICKINSON, J. L. et al. The current state of citizen science as a tool for ecological research and public engagement. Frontiers in Ecology and the Environment, v. 10, n. 6, p. 291-297, 2012.

FEARNSIDE, P. M. The rate and extent of deforestation in Brazilian Amazonia. Environmental Conservation, v. 17, n. 3, p. 213-226, 1990.

FORMAN, R. T. T.; ALEXANDER, L. E. Roads and their major ecological effects. Annual review of ecology and systematics, v. 29, n. 1, p. 207-231, 1998.

GARDINER, M. M. et al. Lessons from lady beetles: accuracy of monitoring data from US and UK citizen-science programs. Frontiers in Ecology and the Environment, v. 10, n. 9, p. 471-476, 2012.

GRAY, S. et al. Combining participatory modelling and citizen science to support volunteer conservation action. Biological conservation, v. 208, p. 76-86, 2017.

GRILO, Clara et al. BRAZIL ROAD-KILL: a data set of wildlife terrestrial vertebrate road-kills. Ecology, v. 99 , n. 11, p. 2625-2625, 2018.

HEIGL, F. et al. Comparing road-kill datasets from hunters and citizen scientists in a landscape context. Remote Sensing, v. 8, n. 10, p. 832, 2016.

JAEGER, J. A. G. Improving environmental impact assessment and road planning at the landscape scale. Handbook of road ecology, p. 32-42, 2015.

KOBORI, H. et al. Citizen science: a new approach to advance ecology, education, and conservation. Ecological research, v. 31, n. 1, p. 1-19, 2016.

LAURANCE, W. F.; GOOSEM, M.; LAURANCE, S. G. W. Impacts of roads and linear clearings on tropical forests. Trends in Ecology \& Evolution, v. 24, n. 12, p. 659-669, 2009.

MONGE-NÁJERA, J.; SEAS, C.. Citizen science and roadkills: trends along project lifespan and comparison of tropical and temperate projects. UNED Research Journal, v. 10, n. 1, 2018.

NOVELLI, R.; TAKASE, E.; CASTRO, V. Estudo das aves mortas por atropelamento em um trecho da rodovia BR-471, entre os distritos da Quinta e Taim, Rio Grande do Sul, Brasil. Revista Brasileira de Zoologia, v. 5, n. 3, p. 441-454, 1988.

OLSON, D. D. et al. Monitoring wildlife-vehicle collisions in the information age: how smartphones can improve data collection. PloS one, v. 9, n. 6, p. e98613, 2014.

PERZ, S. G. et al. Unofficial road building in the Amazon: socioeconomic and biophysical explanations. Development and Change, v. 38, n. 3, p. 529-551, 2007.

PÉRIQUET, S. et al. Testing the value of citizen science for roadkill studies: a case study from South Africa. Frontiers in Ecology and Evolution, v. 6, p. 15, 2018.

PINTO, F. A. S. et al. Giant anteater (Myrmecophaga tridactyla) conservation in Brazil: Analysing the relative effects of fragmentation and mortality due to roads. Biological Conservation, v. 228, p. 148-157, 2018. 
VAN DER REE, R. et al. Effects of roads and traffic on wildlife populations and landscape function: road ecology is moving toward larger scales. v. 16, n. 1, 2011.

VERCAYIE, D.; HERREMANS, M. Citizen science and smartphones take roadkill monitoring to the next level. Nature Conservation, v. 11, p. 29, 2015. WILSON, S. et al. Citizen science reveals an extensive shift in the winter distribution of migratory western grebes. PLoS One, v. 8, n. 6, p. e65408, 2013. 